\title{
The study of the influence of the rock mass heterogeneity on the stress-strain state of the shaft lining
}

\author{
Alexander Pankratenko ${ }^{1, *}$ and Alexander Isaev $^{2}$ \\ ${ }^{1}$ National University of Science and Technology MISIS (Moscow Institute of Steel and Alloys), \\ 119991, Leninskij Av., 4, Moskow, Russia \\ ${ }^{2}$ VostokCoal Management Company, 143084, estate 100, "Residence on Rublevka" Business Center, \\ Usovo, Odinstovsky District, Moscow Region, Russia
}

\begin{abstract}
Shafts often cross sections of a layered mass of rocks of different strength during the sinking. The article deals with the design case of interaction of monolithic concrete lining in the bottomhole zone of the shaft with a mass containing a rock layer with low stress-strain properties. To study its influence, spatial numerical models have been developed, which allowed us to consider several indicative positions of the «weak» layer referred to the shaft lining. The analysis of calculation results shows that in case of direct contact of the support with a «weak» layer, the increase in stress intensity in it can reach 5 -fold values and is determined by the thickness of the «weak» layer and its stress-related characteristics. The most effective control action in this context is the advance hardening of the «weak» layer before its expo-sure during the sinking.
\end{abstract}

\section{Introduction}

The stress strain behavior of vertical well bore lining is determined by a large number of factors. These include: the diameter and depth of the shaft, the stress-related properties of rocks, the parameters of the technological construction scheme, the construction of the lining, etc. Accounting for the geometric characteristics of the shaft can be carried out within the framework of the analysis of two-dimensional grid [1].

The scheme of contact interaction of the system "lining - rock mass" in an elastic approach has wide applicability. The influence of the stage of the initial interaction between the support and the rock mass is made with the help of corrective coefficients. Their exact values are determined on the basis of statistical processing of data monitoring of the bottomhole area, depending on the technological scheme $[2,3]$.

Recognition of the plastic properties of rocks is possible when considering both analytical and numerical models. An ideal-elastic-plastic model with a limiting surface described by the Coulomb-Mohr criterion (the Coulomb-Mohr model) is used [4,5]. At the same time, an analysis of the mining and geological conditions of mining shafts, for example in Donbass (Russia), shows that the rock mass is often represented by areas of intercalation of clay and

\footnotetext{
*Corresponding author: pankrat54@bk.ru
} 
sandy shales, sandstones and limestones with rows and interstratified layers of coal. Layers strength parameters differ by $30 \%$ or more and have a thickness of 0.1 to $5 \mathrm{~m}$.

A number of layers are very disturbed and have very low stress-related properties in comparison with the main rock strata. Studying the stress-strain state of the lining under previously described conditions necessitates the development of spatial numerical models of large dimensions and the implementation of labor-intensive calculations.

\section{Task assignment}

The design case of the interaction of monolithic concrete lining in the bottomhole area of the shaft containing the «weak» rock layer with the following characteristics is considered: the deformation modulus $E_{w l}=1 \cdot 10^{3} \mathrm{MPa}$, the coefficient of lateral deformation $v_{w l}=0.4$. The thickness of the «weak» layer varied in the range $m=1-5 \mathrm{~m}$, the angle $\alpha=0-45^{\circ}$.

The remaining parameters of the models and ranges of their variation are taken as averaged for engineering and geological conditions of the Donbass.

Three characteristic positions of the «weak» layer in the bottomhole are of the shaft were singled out during the investigation (Fig. 1). For each position, a three-dimensional finiteelement model has been developed for the LIRA CAD program. As a result all the components of the strain and stress tensor were determined by varying the initial data.

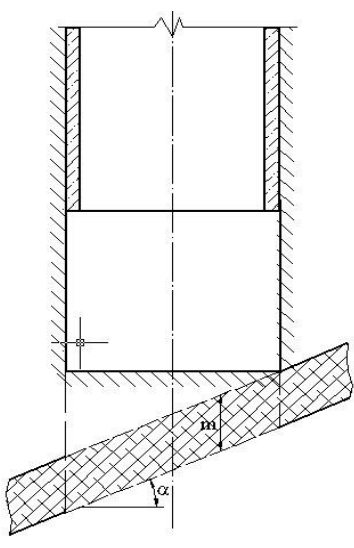

a

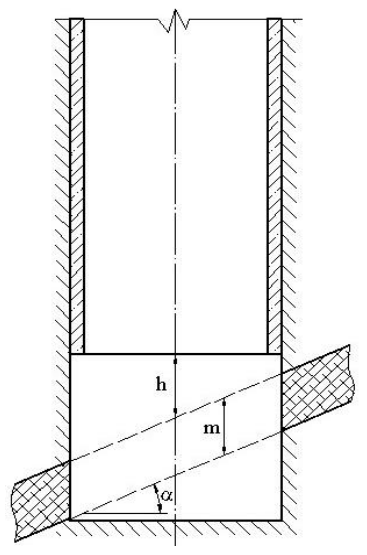

b

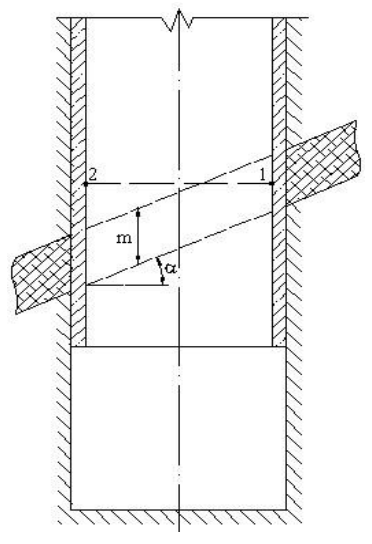

c

Fig. 1. Scheme of design case of placing a «weak» layer in the bottomhole area of the shaft.

The models had a spatial prismatic shape. The height of the models was $100 \mathrm{~m}$, the face size was assumed equal to $10 d$, where $d$ is the diameter of the shaft in clear.

Elements of the lining and rocks mass were modeled with the help of spatial isoparametric eight-node finite elements, which make it possible to determine the parameters of the stressstrain state, both continual objects and massive structures.

The boundary conditions of the models are given in the form of restrictions on the movement of the nodes of the outer cylindrical face of the rock mass of the model in the perpendicular direction. Evenly distributed loads from the rock pressure were applied to the top of the model. This approach is widely approved.

\section{Analysis and interpretation of research results}

Analysis of the stress-strain state of the models showed that the presence of a «weak» layer leads to the occurrence of concentrations of the main horizontal stresses in the adjacent 
sections of stronger rocks, and also increases the intensity of radial displacements on the shaft contour and loads on the lining in the area of the «weak» layer.

To estimate the quantitative effect of the «weak» layer on the stresses in the monolithic concrete lining, the parameter $\sigma_{r e l}$ is introduced. It is the ratio of the maximum principal stresses in the lining in the zone of the «weak» layer to the analogous values obtained in calculating the model in a homogeneous rock mass.

It was found that when the «weak» layer is in position 1 (Fig. 1a), its influence on the intensity of stresses in the lining close to the borehole bottom is insignificant. The increase in stresses in the lining is not more than $4-5 \%$ and is practically independent of the thickness of the «weak» layer, the ratio $G_{0} / G_{w l}$, where $G_{0}$ is the shift modulus of the main rock mass, $G_{w l}$ is the modulus of shift of the «weak» layer rocks, and other system parameters.

In position 2, the influence of the «weak» layer on the shaft supports significantly increases. In Fig. 2 you can see the dependence of $\sigma_{\text {rel }}$ on the distance $h$ of the cross-section of the concrete lining to the upper section of the «weak» layer (see Fig. 1b). The graph are constructed from the data obtained at a depth of $500 \mathrm{~m}$, the deformation module of the main rock mass $15 \cdot 10^{3} \mathrm{MPa}$.

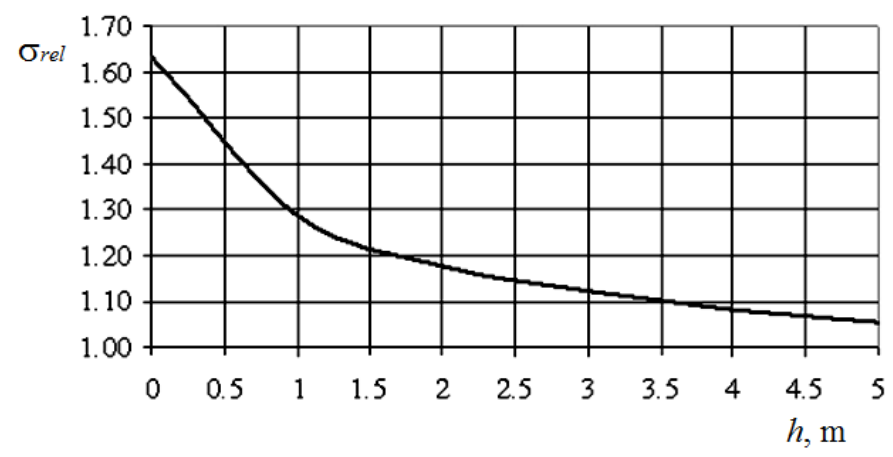

Fig. 2. Dependence of the parameter $\sigma_{\text {rel }}$ on distance

Similar data was obtained under the application of other parameters of numerical models.

When the «weak» layer is directly contacts with the fixture (Fig. 1c), a significant increase in the parameter $\sigma_{\text {rel }}$ is observed. The deformation of the bend takes place in the lining, leading to the formation of tensile stress zones, abnormal for a homogeneous mass.

The dependences of the parameter $\sigma_{r e l}$ on the thickness of the «weak» layer and the ratio $G_{0} / G_{w l}$ are shown in Fig. 3 and 4.

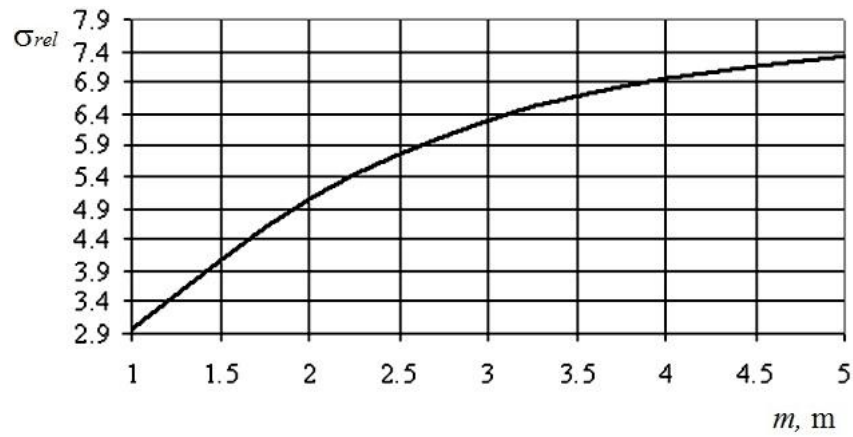

Fig. 3. Dependence of the parameter ${ }_{-}^{-}$rel on the thickness of the «weak» layer in position c (see Fig. $1 \mathrm{c})$. 


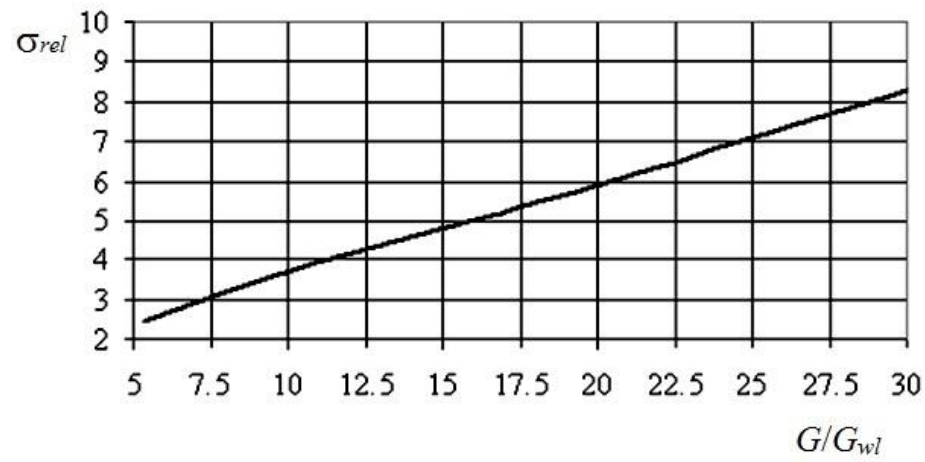

Fig. 4. Dependence of the parameter $\sigma_{\text {rel }}$ on the ratio $G_{0} / G_{w l}$.

Data processing shows that when the «weak» layer in position 3 is in contact with the monolithic lining, the intensity of maximum stresses increases: when the thickness of the «weak» layer increases, the parabolic dependence increases, as the ratio $G_{0} / G_{w l} l$ increases with respect to the linear dependence.

When the monolithic concrete lining interacts with a «weak» layer having an inclined bedding, a significant asymmetry of the main tangential stresses occurs with the formation of local coordination areas, as well as an increase in stretching radial stresses in the support rings adjacent to the «weak» layer. The coefficient of unevenness $k_{u n}$ was introduced to quantify the magnitude of the stress asymmetry

$$
k_{u n}=\frac{\sigma_{11}}{\sigma_{12}}
$$

where $\sigma_{11}$ is the magnitude of the maximum stresses in the monolithic concrete lining at point 1 (see Fig. 1c);

$\sigma_{12}$ is the magnitude of the maximum stresses in the monolithic concrete lining at point 2 (see Fig. 1c) located at the same height as the point 1 with the opposite side of the section of the shaft relative to the fall of the «weak» layer.

Fig. 5 shows the obtained dependence of the coefficient $k_{u n}$ on the incidence angle of the «weak» layer $\alpha$, with the layer thickness $m=1 \mathrm{~m}$ and $m=3 \mathrm{~m}$. The deformation modulus of the main rock mass was $15 \cdot 10^{3} \mathrm{MPa}$.

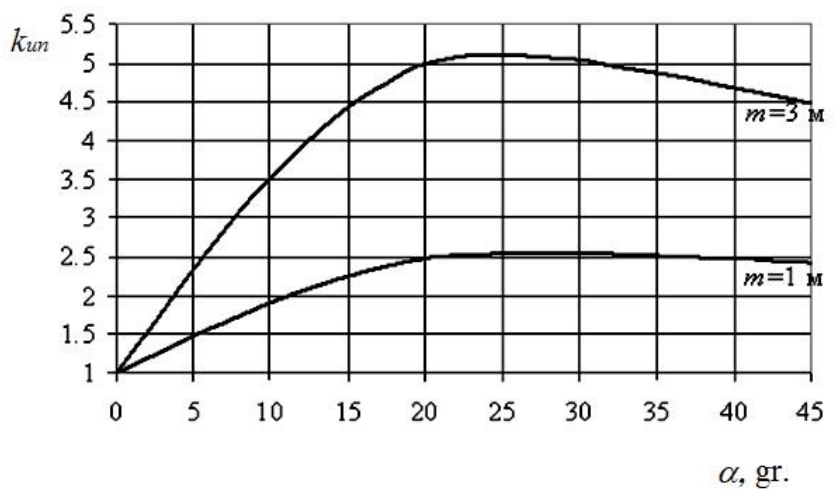

Fig. 5. Dependence of the coefficient $k_{u n}$ on the bedding angle of the «weak» layer.

Analysis of the data shows that in the range $\alpha=0-25^{\circ}$, the asymmetry of the stresses in the lining section increases according to the hyperbolic dependence, with the maximum attained 
at $\alpha=25^{0}-30^{\circ}$, then a slight decrease in the values of the coefficient $k_{u n}$ is observed followed by their equalization.

\section{Results}

The main results of the study are as follows:

1. In position 1, the «weak» layer does not significantly affect the stress-strain state of the monolithic concrete lining, regardless of its power, angle of incidence and other parameters.

2. In position 2 , it is possible to increase the intensity of stresses in the lining in 1.5-2 times or more, increasing with thickness of the layer and decreasing the distance from it to the lower edge of the concrete lining.

3. In the case of direct contact of the support with a «weak» layer, the increase in stress intensity in the lining can reach 5-fold values and is determined by the thickness of the «weak» layer and the ratio $G_{0} / G_{w l}$. The change in the formation angle does not lead to the increase in the maximum stresses in the lining, but increases the size of the influence area of the «weak» layer and causes a significant asymmetry in the distribution of stresses in the support ring.

From the results of the research it follows that the most effective control actions aimed at reducing the influence of the «weak» layer on the shaft lining is the advance hardening of the «weak» layer prior to its exposure in the process of sinking, as well as parts of stronger rocks in contact with it and, if necessary, the lining itself.

\section{References}

1. F.I. Yagodkin, A.Y. Prokopov, M.S. Pleshko, A.N. Pankratenko, IOP Conference Series: Earth and Environmental Science, 87(6), 062014 (2017)

2. F. Yagodkin, M. Pleshko, A. Prokopov, Procedia Engineering, 206, 293-298 (2017)

3. M. Pleshko, B. Meskhi, M. Pleshko, MATEC Web of Conferences, 170, 03023 (2018)

4. B. Meskhi, M. Pleshko, Y. Buligin, L. Alexeenko, M. Molev, IOP Conference Series: Earth and Environmental Science, 90(1), 012217 (2017)

5. A. Pankratenko, M. Pleshko and A. Isaev, MATEC Web of Conferences, 7, 02026 (2018) 